journal club

\title{
Teil- oder vollstationär behandeln?
}

Fragestellung: Ist das teilstationäre dem vollstationären Therapie-Setting bei der adoleszenten Anorexia nervosa unterlegen?

Hintergrund: Das vollstationäre Setting ist bei mittel- bis schwerkranken Patienten mit Anorexia nervosa die Behandlung der Wahl. Jedoch treten Rückfälle und ein damit verbundener hoher individueller Leidensdruck häufig auf. Zudem verursacht die stationäre Behandlung hohe Kosten. Eine Pilotstudie deutete darauf hin, dass eine teilstationäre Behandlung bei der jugendlichen Anorexia nervosa die Rückfallrate verringern könnte.

Patienten und Methodik: Im Rahmen der multizentrischen, randomisierten, kontrollierten Nicht-Unterlegensheitsstudie erhielten 85 Patientinnen eine vollstationäre und 87 eine teilstationäre Behandlung (Alter 11 bis 18 Jahre). In beiden Studienarmen erfolgte vor der differenziellen Behandlung eine dreiwöchige vollstationäre Stabilisierung und im Anschluss eine ambulante Nachsorge. Für beide Herpertz-Dahlmann B, Schwarte R, Krei M et al. Daypatient treatment after short inpatient care versus continued inpatient treatment in adolescents with anorexia nervosa (ANDI): a multicentre, randomised, open-label, non-inferiority trial. Lancet 2014; 383: $1222-9$ den die Patientinnen entlassen, sobald sie ihr Zielgewicht erreicht hatten und zwei Wochen hielten (15. bis 20. Perzentile). Primärer Endpunkt war die BMI-Differenz zwischen Aufnahme und Follow-up-Untersuchung nach einem Jahr oder der Wiederaufnahme korrigiert für Alter und Krankheitsdauer. Sekundäre Endpunkte waren die Psychopathologie mittels Average MorganRussell-Kriterium, Eating Disorder Inventory II und Brief Symptom Inventory, die Häufigkeit von stationären Aufenthalten über den gesamten Beobachtungszeitraum von 52 Wochen ab Aufnahme und der Unterschied der Krankenkassenkosten.

Ergebnisse: Die teilstationäre war der stationären Behandlung hinsichtlich des primären Endpunktes nicht unterlegen. Die teilstationäre Behandlung verursachte $20 \%$ weniger Kosten als die vollstationäre Therapie. Es zeigte sich kein signifikanter Unterschied bezüglich des General Morgan and Russell Scores und der Wiederaufnahmen zwischen den Gruppen, jedoch erschienen mehr Patientinnen im stationären Behandlungsarm nicht zu den Nachuntersuchungen. Die Anzahl behandlungsabhängiger unerwünschter Ereignisse war in beiden Gruppen ähnlich. Drei Patientinnen in der stationären und zwei in der teilstationären Gruppe entwickelten Suizidgedanken. Eine Patientin in der teilstationären Gruppe suizidierte sich drei Monate nach Entlassung.

Schlussfolgerungen: Die teilstationäre war der stationären Behandlung bei Patientinnen mit Anorexia nervosa nicht unterlegen. Die Therapiekosten waren im tagesklinischen Setting niedriger.

\section{- Kommentar von Polyxeni Bouna-Pyrrou, Erlangen}

\section{Die teilstationäre ist der stationären Behandlung nicht unterlegen}

Es handelt sich um eine der wenigen, wenn nicht die einzige Studie, die eine gestufte teilstationäre Behandlung mit einer vollstationären Behandlung bei Patientinnen mit Anorexia nervosa vergleicht. Die Studie erfüllt mit einem JADAD-Score von vier Punkten hohe methodische Ansprüche. Sie weist nach, dass die teilstationäre der stationären Behandlung hinsichtlich der Wirksamkeit nicht unterlegen ist. Die beiden Studien-Settings waren vergleichbar, es handelte sich um ein identisches Therapieprogramm mit denselben Therapeuten in beiden Studienarmen. Hervorzuheben ist die niedrige Abbrecherquote, 161 der 172 Teilnehmer (94\%) absolvierten das erste Assessment nach zwölf Monaten. Die klinische Relevanz der Studie ist hoch, es wurde eine große repräsentative Stichprobe untersucht und die Patientinnen wurden über einen Zeitraum von zwölf Monaten beobachtet.

Einschränkend ist zu bemerken, dass die Ergebnisse der Studie womöglich eher bei jungen Patientinnen mit einer kurzen Krankheitsdauer replizierbar sind. Problematisch ist zudem die Berechnung der Kosten. Es wurden nur direkte Krankenkassenkosten bewertet und keine Kosten, die aus Zusatzbehand- lungen (z. B. ambulante Behandlung) oder Transport entstanden sind. Anzuregen wäre die Erweiterung der Follow-upUntersuchungen über den Zeitraum von zwölf Monaten.

Die Ergebnisse dieser Studie rechtfertigen insgesamt die flächendeckende Implementierung der teilstationären Behandlung in der klinischen Regelversorgung von adoleszenten Patientinnen mit Anorexia nervosa.

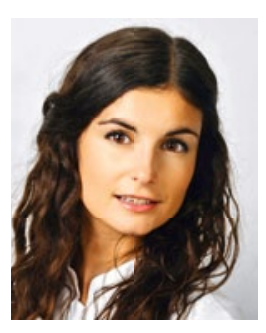

Polyxeni Bouna-Pyrrou, Erlangen

Klinik für Psychiatrie und Psychotherapie Universitätsklinikum Erlangen

E-Mail: polyxeni.bouna-pyrrou@uk-erlangen.de 